\title{
Parametric Models of Thermal Transfer Impedances within a Successive Node Reduction Based Thermal Simulation Environment
}

\author{
Márton Németh ${ }^{1 *}$, András Poppe ${ }^{1}$ \\ ${ }^{1}$ Department of Electron Devices, Faculty of Electrical Engineering and Informatics, Budapest University of Technology and \\ Economics, H-1117 Budapest, Magyar tudósok krt. 2, Hungary \\ ${ }^{*}$ Corresponding author, email: nemeth@eet.bme.hu
}

Received: 20 May 2017, Accepted: 15 January 2018, Published online: 15 February 2018

\begin{abstract}
In this paper we present a new, direct computational method for calculating thermal transfer impedances between two separate locations of a given physical structure, aimed at the implementation into a field-solver based on the SUNRED (SUccessive Node REDuction) algorithm. We tested the method symbolically with a simple 2D example with multiple combinations of Dirichlet and Neumann type boundary conditions. Also, for time domain transient analysis different types of thermal loads such as prescribed unit-step change in dissipation or temperature were assumed. A model of a typical MCPCB assembled LED was also created. With that model we studied the inverse problem of predicting the thermal conditions at the junction (the "driving point") from the transient signal measured at the thermal test point on the MCPCB (the "monitoring point") which is a typical task in simple LED thermal management designs. Results obtained by the proposed new calculation method and results obtained by conventional numerical simulations differ less than the uncertainty of the traditional solution method itself. The drawback of the accuracy is the high computational cost. This increased computational need can be mitigated by introducing the combination of the balanced model reduction and the SUNRED algorithms.
\end{abstract}

Keywords

compact thermal modeling, thermal transfer functions, node reduction, inverse problem

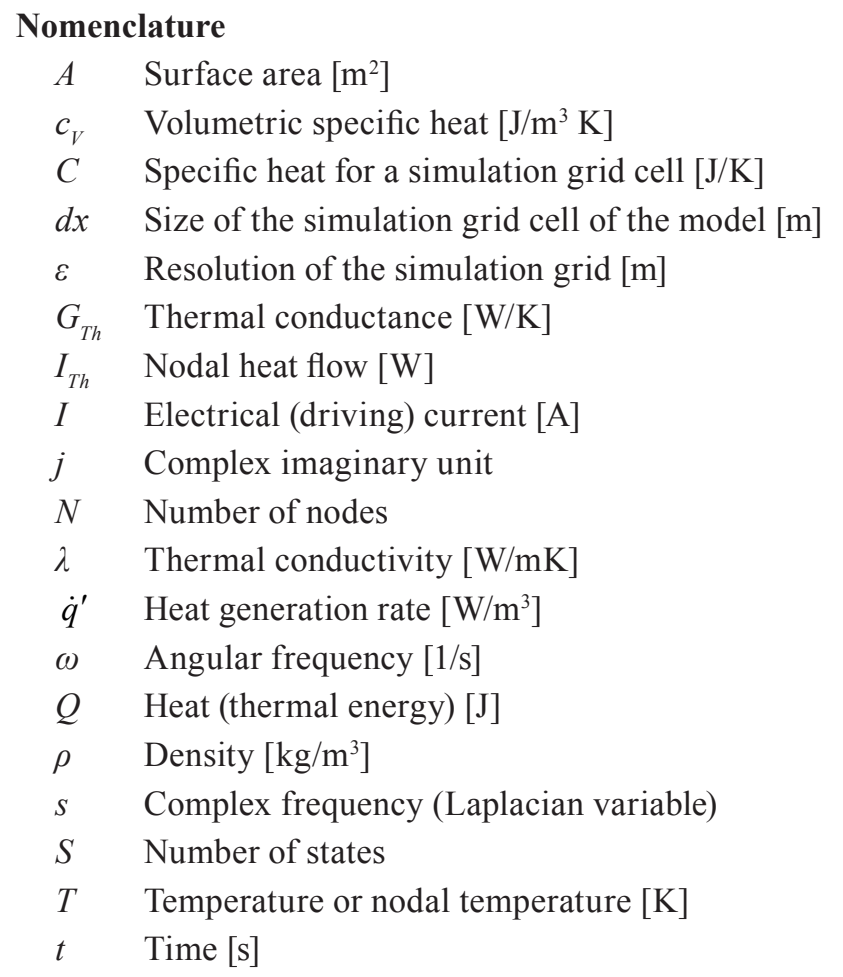

$\begin{array}{ll}U & \text { Electrical (driving) voltage }[\mathrm{V}] \\ \underline{Y} & \text { Admittance matrix }\end{array}$

1 Introduction

Nowadays we face a growing interest in more accurate thermal modeling of electronic parts, such as ICs, discrete power semiconductor devices, system-in-package (SiP) devices and LEDs. In the past two decades the need for higher speed of numerical simulations and the fact that semiconductor vendors did not want to share proprietary information about their advanced packaging solution resulted in the development of compact thermal models of the packages of electronic components. Such compact thermalmodels represent the thermal behavior of the package by means of a set of lumped thermal resistances and capacitances. In his 2008 paper [1] Clemens J. M. Lasance provided an overview of the state of the art at that time. From the known methods of that time the so 
called JEDEC 2R model (a very simplistic model) and the DELPHI model topology and modeling methodology became an industry standard [2-4]. For a given IC package the DELPHI compact modeling methodology uses a global optimization method to find the network element values of the fixed DELPHI model topology which fit the simulation results for a great variety of different thermal boundary conditions obtained by CFD simulations (using the detailed geometrical model of the package) the best ${ }^{1}$. Nowadays such detailed simulation models are validated or even automatically calibrated against physical measurement results using the method of structure function analysis [5].

The major problem with the DELPHI compact models is the fixed topology and e.g. despite some efforts aimed at modeling stacked die IC packages [6] the lack of ability of describing multi-heat source packages. Another problem of the DELPHI model is that it is a steady-state model. Within the European project PROFIT [7] a global optimization method was used to generate boundary condition independent dynamic compact thermal models for single-source IC-packages with multi-directional heat flow pattern [8]. In the PROFIT approach first a steadystate thermal resistance network is generated which is then extended to a dynamic model by adding appropriate thermal capacitances. D. Schweitzer provided a detailed overview of the mathematical description of multi-heat source problems $[9,10]$. Though in this paper D. Schweitzer provides a general formulation of the problem and he established a terminology that we also try to follow in our present work, no mentioning of a compact thermal modeling methodology is given which is aimed at the description of multi heat-source problems with any geometrical arrangement and is not restricted to semiconductor device packages. Inspired by this paper as well as by our prior work in the field of electrothermal simulation of analog ICs given by their layout [11], we recently reported about a systematic approach of creating a steady-state compact thermal model of an LED based streetlighting luminaire $[12,13]$. In the meanwhile, L. Codecasa et al. $[14,15]$ reported on model order reduction methods (implemented in an academic software tool [16]) which are aimed for creating dynamic compact thermal

1 The "goodness" of the fit of the obtained compact thermal model for the different thermal boundary conditions is described by the so-called boundary condition independence index (also known as BCI index). The goal is to have the highest degree of boundary condition independence to allow the compact thermal model represents the part in any thermal environment. models of multi heat-source systems described by any kind of detailed geometry.

The advantage of these models (like that of any previous compact thermal models) is that the proprietary information of the system is hidden from the users; in these new approaches it is coded into transfer functions, which can be instantly used for transient or steadystate simulation. The parametric analysis feature of these new methods is very useful, when there are parts in the structure which can be varied in size or material [17]. In this paper we also apply this parametricanalysis techniques in the symbolic calculations.

The order reduction process of such thermal systems represented by their detailed numerical models can be based on projection to the Krylov-subspace (as described e.g. in [18]). These methods are extremely efficient, however there is no theory established which assures that the resulting models are optimal [19]. As an alternative to the above mentioned projection based methods, the balanced model reduction can be used, but its very high computational cost limits its use, especially on complex (high ordered) systems. This problem however, can be overcome with the combination of the traditional successive node reduction (SUNRED) and the balanced order reduction algorithms. While the prior limits the number of nodes, thelatter limits the order of the transfer functions that represent the complex thermal impedances between two arbitrary chosen nodes of the system.

\section{Modeling}

\subsection{The SUNRED algorithm}

The SUNRED algorithm is centered around the admittance matrix of the entire electrical equivalent circuit derived from the space discretized form of the thermal diffusion equation $[20,21]$ :

$c_{V} \frac{\partial T}{\partial t}-\nabla(-\lambda \nabla T)=\dot{q}^{\prime}(\underline{r}, t)$

The discretization is done in space and time:

$$
\begin{array}{r}
\frac{\lambda}{\varepsilon^{2}}\left(T_{i+1, j}+T_{i-1, j}+T_{i, j+1}+T_{i, j-1}-4 T_{i j}\right) \\
=P+c_{V} \frac{T_{i, j}(t)+T_{i, j}(t-d t)}{d t}
\end{array}
$$

This algebraic equation system for the time domain propagation can be represented also as a matrix-equation:

$\underline{T}(t+d t)=\underline{T}(t)+d t \cdot \underline{Y} \underline{T}$ 
For the sake of simplicity in setting up the thermal admittance matrix $\underline{Y}$ we use the thermal conductance:

$$
G_{T h}=\frac{Q_{\text {through }}}{\Delta T}=\frac{\lambda A}{d x}=\lambda d x
$$

where an equidistant discretization scheme was assumed. With the help of the above thermal conductivity, the admittance matrix can be written (in 2D) as follows:

$$
\underline{Y}=\left(\begin{array}{ccccc}
-4 G_{T h} & G_{T h} & G_{T h} & G_{T h} & 0 \cdots 0 \\
G_{T h} & -4 G_{T h} & G_{T h} & \ddots & \vdots \\
G_{T h} & G_{T h} & -4 G_{T h} & \ddots & \vdots \\
\vdots & \ddots & \ddots & \ddots & \vdots \\
\vdots & \ddots & G_{T h} & -4 G_{T h} & G_{T h} \\
G_{T h} & 0 \cdots 0 & G_{T h} & G_{T h} & -4 G_{T h}
\end{array}\right)
$$

where $G_{T h}$ appears in the $\mathrm{i}^{\text {th }}$ row $\mathrm{k}^{\text {th }}$ column, if the node at $(x, y)$ coordinate, which belongs to the row $i$ in the matrix, is connected to another node (i.e. $x, y-1$ ) which is represented by column $k$. The other elements of the matrix are zero, indicating the lack of direct connection between the represented nodes. With this matrix one can determine the heat fluxes between nodes by applying Kirchhoff's laws:

$\underline{I}_{T h}=\underline{Y} \underline{T}+\underline{I}_{g e n}$

where $\underline{I}_{T h} \underline{T}$ and $\underline{I}_{\text {gen }}$ are vectors with $N$ element that represent each node. The steady-state thermal solution can be derived from Eq. (6) with the assumption of $\underline{I}_{T h}=0$ :

$$
\underline{T}=-\underline{Y}^{-1} \underline{I}_{g e n}
$$

The admittance matrix can be reduced by merging the neighboring nodes with the so-called successive node reduction (SUNRED) algorithm.

To implement the resistive equivalent model of the conductive heat transfer in the SUNRED algorithm, first one has to determine the appropriate admittance matrix of such a system. The basic building block in the SUNRED algorithm (representing a cube shaped simulation grid cell) is a circuit cell with four external points (see Fig. 1 (b)). Such an elementary building block is used to represent an original node with terminals that connect it to other similar building blocks. Originally, all nodes are connected to each other by the resistive element with thermal conductivity $G_{T h} ; 0$ conductivity means that there is no connection between two nodes, thus the admittance matrix of a basic building block can be written as:

$$
\underline{Y}=\left(\begin{array}{ccccc}
-G_{T h} & 0 & 0 & 0 & G_{T h} \\
0 & -G_{T h} & 0 & 0 & G_{T h} \\
0 & 0 & -G_{T h} & 0 & G_{T h} \\
0 & 0 & 0 & -G_{T h} & G_{T h} \\
G_{T h} & G_{T h} & G_{T h} & G_{T h} & -4 G_{T h}
\end{array}\right) .
$$
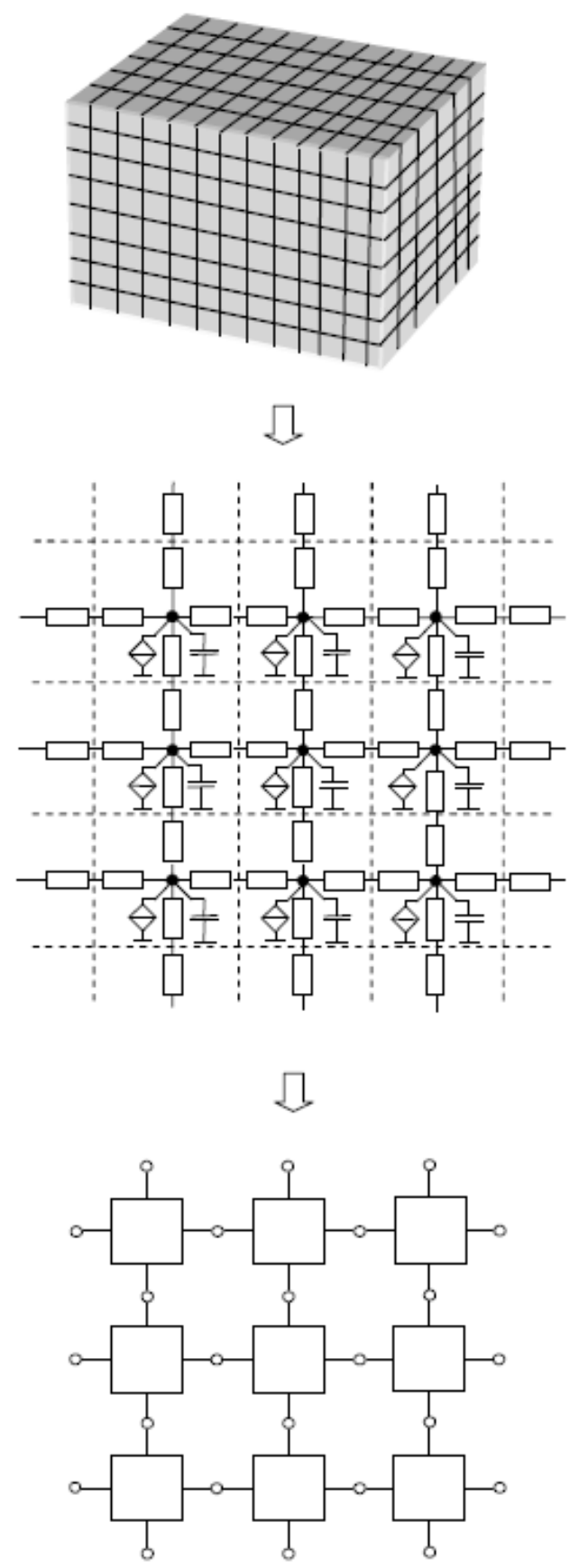

Fig. 1 SUNRED algorithm a) 3D rectangular field b) SUNRED version of Finite Differences model in 2D c) The model after the first node reduction step (elimination of the internal nodes of the first level cells) [22] 
This represents one of the four building blocks in Fig. 2 . The rows and columns of 1-4 represent the connections of the nodes on the sides of the rectangle, while the fifth row and column represents the central node's connections of a standalone building block.

After all the connections for the central node are established, the node can be eliminated. The elimination process (resembling the classical an Y- $\Delta$ transformation) in matrix form looks as follows:

$\underline{\underline{Y}}_{\text {red }}=\underline{\underline{Y}}_{\text {outer }}-\underline{\underline{Y}}_{\text {in-out }} \underline{\underline{Y}}_{\text {innner }}^{-1} \underline{\underline{Y}}_{\text {out }- \text { in }}$

where $Y_{\text {red }}$ is the reduced admittance matrix, $Y_{\text {outer }}$ is the matrix of the connections only from the maintained nodes (in the example the rows and columns form 1 to 4), $Y_{\text {in - out }}$ contains the connection between the inner and outer nodes (fifth row 1-4 columns), as the $Y_{\text {out - in }}$ (fifth column 1-4 rows) and $Y_{\text {inner }}$ stands for the inner node connections (fifth column and row). Therefore, the reduced matrix of the building block is:

$\underline{Y}_{r e d}=\frac{G_{T h}}{4}\left(\begin{array}{cccc}-3 & 1 & 1 & 1 \\ 1 & -3 & 1 & 1 \\ 1 & 1 & -3 & 1 \\ 1 & 1 & 1 & -3\end{array}\right)$

The connection between the building blocks can be established by the expansion of the matrix by the new nodes, as if the connected nodes would be different. The joint nodes are connected with the nodes of both blocks, so that they can be handled with the sum of the representing rows and columns of both representation. After the connection, the node can be eliminated, resulting in the reduction of the order of the admittance matrix.

In case of transient simulations, the capacitors representing the thermal capacitance of a grid cell are replaced with their time-discretized resistive equivalent corresponding to the actual time-domain numerical solution method. Basically, such an equivalent represents a capacitor with a resistor and with a "current" source. In case of thermal capacitance the source value is equal to the heat-flux resulting from the change of the thermal energy stored in the volume of material represented by the simulation grid cell during the given simulation time interval $\Delta t$. The value of the "current" depends on this $\Delta t$ simulation time-step (see Fig. 3) therefore the whole temperature-map of the original system must be recalculated for the subsequent simulation time-steps.

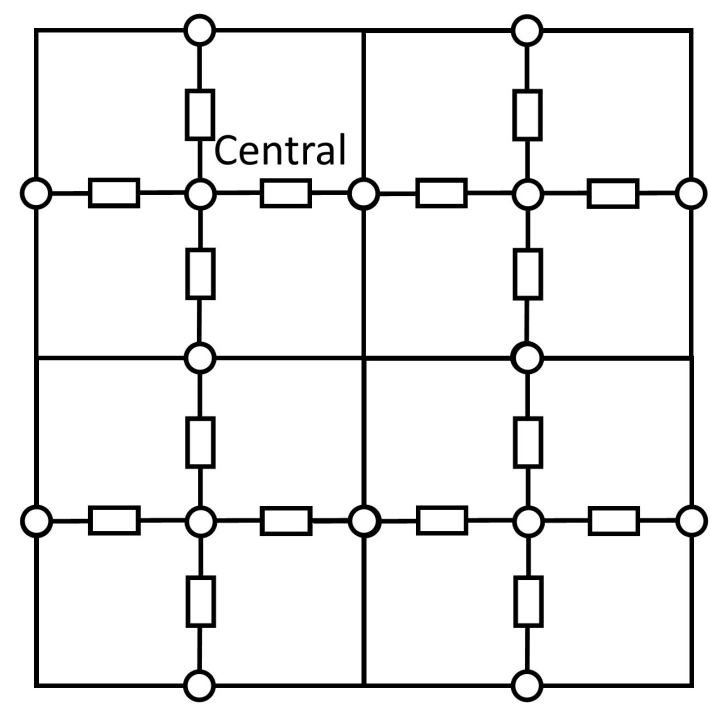

Fig. 2 Four 2D building blocks in a network

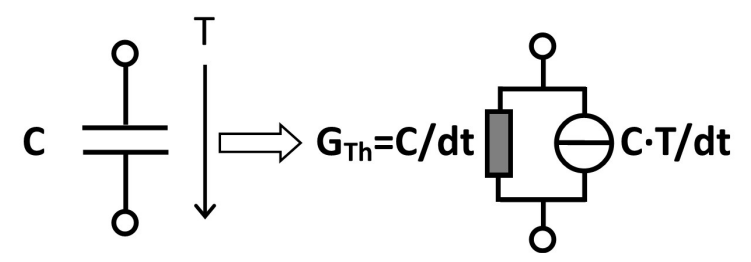

Fig. 3 The time discredized resistive equivalent of a capacitor (for the time-domain numerical integration scheme based on the reverse Euler method

\section{SUNRED algorithm with transfer functions representing thermal transfer impedances}

With the Laplace transform of Eq. (2) the SUNRED algorithm can also be formulated for the system behavior in the complex frequency $(s)$ domain as follows:

$$
\begin{aligned}
& \frac{\lambda}{\varepsilon^{2}}\left(T_{i+1, j}(s)+T_{i-1, j}(s)+T_{i, j+1}(s)+T_{i, j-1}(s)-4 T_{i j}(s)\right) \\
& =P+c_{V} s T_{i j}(s)
\end{aligned}
$$

This means that the admittance matrix itself depends on the complex frequency $s$ (in the Laplace domain), therefore the building blocks need to be modified as follows:

$$
\underline{Y}=\left(\begin{array}{ccccc}
-G_{T h} & 0 & 0 & 0 & G_{T h} \\
0 & -G_{T h} & 0 & 0 & G_{T h} \\
0 & 0 & -G_{T h} & 0 & G_{T h} \\
0 & 0 & 0 & -G_{T h} & G_{T h} \\
G_{T h} & G_{T h} & G_{T h} & G_{T h} & -4 G_{T h}-C S
\end{array}\right)
$$

where $C$ stands for the thermal capacitance of the whole block, as shown in Fig. 4. The reduction algorithm is the 


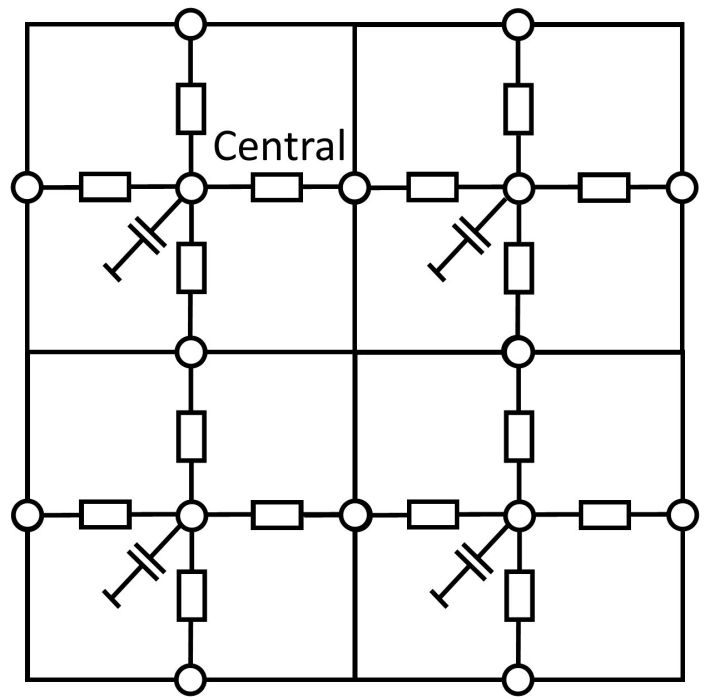

Fig. 4 Four two dimensional building blocks with capacitor in a network

same as outlined above, therefore the reduced building block can be represented with the following equation:

$$
\begin{aligned}
& \underline{\underline{r}}_{\mathrm{red}}= \\
& =\left(\begin{array}{llll}
\frac{G_{T h}^{2}}{4 G_{T h}-C s}-G_{T h} & \frac{G_{T h}^{2}}{4 G_{T h}-C s} & \frac{G_{T h}^{2}}{4 G_{T h}-C s} & \frac{G_{T h}^{2}}{4 G_{T h}-C s} \\
\frac{G_{T h}^{2}}{4 G_{T h}-C s} & \frac{G_{T h}^{2}}{4 G_{T h}-C s}-G_{T h} & \frac{G_{T h}^{2}}{4 G_{T h}-C s} & \frac{G_{T h}^{2}}{4 G_{T h}-C s} \\
\frac{G_{T h}^{2}}{4 G_{T h}-C s} & \frac{G_{T h}^{2}}{4 G_{T h}-C s} & \frac{G_{T h}^{2}}{4 G_{T h}-C s}-G_{T h} & \frac{G_{T h}^{2}}{4 G_{T h}-C s} \\
\frac{G_{T h}^{2}}{4 G_{T h}-C s} & \frac{G_{T h}^{2}}{4 G_{T h}-C s} & \frac{G_{T h}^{2}}{4 G_{T h}-C s} & \frac{G_{T h}^{2}}{4 G_{T h}-C s}-G_{T h}
\end{array}\right)
\end{aligned}
$$

Merging the nodes between blocks and the standard node elimination of the successive node reduction method require the same algebraic operation as in the ordinary SUNRED algorithm [23]. The only difference is that now one has to cope with transfer functions.

We can appoint some nodes that will not be eliminated and so we can prescribe the value of the heat flux through such a node or we can prescribe temperature of such nodes. Following the terminology introduced by D. Schweitzer $[9$, 10], let us call such nodes as driving points and/ or monitoring points. When a node is a driving point, it represents a time variant heat dissipating element. In the network representation of the discretized model such a node is driven by a "current source". Monitoring points are locations where we would like to know e.g. the time evolution of the temperature while the state of other such nodes is changing. A node can be both a driving point and a monitoring point. The number of driving points in a system is equal to the number of elementary heat-sources we have in the system. Since any location in the system can be a monitoring point, in a general case the number of monitoring points can be greater or equal to the number of driving points [10]. The "thermal coupling" (or heat propagation) between a driving point and a monitoring point is described by the thermal transfer impedance between these points. The goal of our present study is to provide an efficient method of modelling such thermal transfer impedances which is outlined in the following subsection.

When the reduction process ends the admittance matrix is an $n$ times $n$ matrix of transfer functions, where $n$ is the number of the nodes considered as driving/monitoring points. These transfer functions represent the propagation properties between pairs of driving and monitoring points. To extend the terminology to the transfer functions, the node at the "input" side is called driving point (regardless if it is really driven by a heat-source), the node at the "output" side is a monitoring point. Because of the new nonnumerical parameter $(s)$ in the node reduction process, the thermal resistances and capacitances can also be handled symbolically. In other words, a symbolic transfer function can be created which represents the thermal transfer impedance between two selected nodes of the discretized model of the physical structure (geometry, set of boundary conditions). Changes in materials used in the system can be easily applied through changing the resistance and capacitance values accordingly,without the need of performing the timeconsuming node reduction algorithm again.

The possible distinction between driving and monitoring points is justified by the fact that in practice (during thermal transient measurements of multi heatsource packages) non-reciprocal effects are experienced, see e.g. $[6,24]$. On the level of the discretized model of a system, however, where every heat-source and every monitoring location is point-like, the network model is completely reciprocal, therefore distinction in naming the two nodes associated with "input" and "output" side of a thermal transfer impedance (transfer function) is not necessary, we simply call these terminal nodes as ports. (Distinction between driving point thermal impedances and thermal transfer impedances though, is important and justified, see further explanation e.g. in [25].) In circuit theory, the linear reciprocal networks represented by the above described transfer functions are also known as two ports; reciprocity means that the roles of the ports (i.e. the terminal nodes) are interchangeable. 


\section{Balanced model reduction}

The admittance matrix naturally includes transfer impedances between nodes as off-diagonal elements. The generic form of these transfer functions in the complex frequency domain is:

$\underline{Y}_{i, j}=\frac{A(s)}{B(s)}=\frac{a_{n} s^{n}+a_{n-1} s^{n-1}+\ldots a_{2} s+a_{1}}{b_{m} s^{m}+b_{m-1} s^{m-1}+\ldots b_{2} s+b_{1}}$,

where $m>n$. Let us switch to state-space representation:

$$
\begin{aligned}
\underline{T}(t) & =\underline{\underline{A}} \cdot \underline{T}(t)+\underline{B I}_{\text {in }}(t) \\
\underline{I}(t) & =\underline{C} \cdot \underline{T}(t)
\end{aligned}
$$

where the elements of $A, B$ and $C$ can be read from the Eq. (14) equation. The controllability and observability Gramian is constructed as:

$$
\begin{aligned}
& W_{c}=\left[B ; A B ; A^{2} B \ldots\right] \\
& W_{o}=\left[C ; C A ; C A^{2} \ldots\right]
\end{aligned}
$$

and so the Hankel-matrix (operator):

$$
H=W_{c} \otimes W_{o}=\left(\begin{array}{cccc}
C B & C A B & C A^{2} B & \ldots \\
C A B & C A^{2} B & C A^{3} B & \ldots \\
C A^{2} B & C A^{3} B & C A^{4} B & \ldots \\
\vdots & \vdots & \vdots & \ddots
\end{array}\right)
$$

The eigenvalues of the Hankel-matrix are called Hankel singular values. The Hankel singular values indicate the "importance" of the state that belongs to the value. A small Hankel value means that a state is not controllable, or it is not observable, or both. This means that a small value indicates weak connection between the ports, therefore it can be eliminated. The order of the complex transfer impedance can be preserved in each step of the SUNRED algorithm, if the highest Hankel singular values are preserved.

The balanced order reduction algorithms have high computational cost: the reduction cost is proportional to $S^{3}$ where $S$ is the number of represented states. In the SUNRED algorithm each node-pair is represented as a transfer function, therefore the computational cost of the matrix-operations is proportional to $N^{2}$ where $N$ is the number of nodes before the reduction.

Therefore the reduction of the order of the transfer impedances is essential, since the total cost of computations is proportional to $S^{3} \times N^{2}$.

\section{Prototype implementation and testing}

\subsection{Simulation setups}

The calculation described in the previous subsection was implemented in Matlab 2014 with the help of Matlab's symbolic toolbox. The test setup contained 25 building blocks, and 125 nodes. Homogenous material distribution was assumed in the simulated physical domain. The new transfer impedance calculation method implemented in the SUNRED algorithm was compared with the timedomain thermal transfer impedance functions obtained by the standard thermal transient simulation method of the conventional SUNRED solver. In both cases the same geometry and the same sets of boundary conditions were applied. The simulated test structure is shown in Fig. 5.

\section{$\mathrm{BC}$ : Dirichlet}
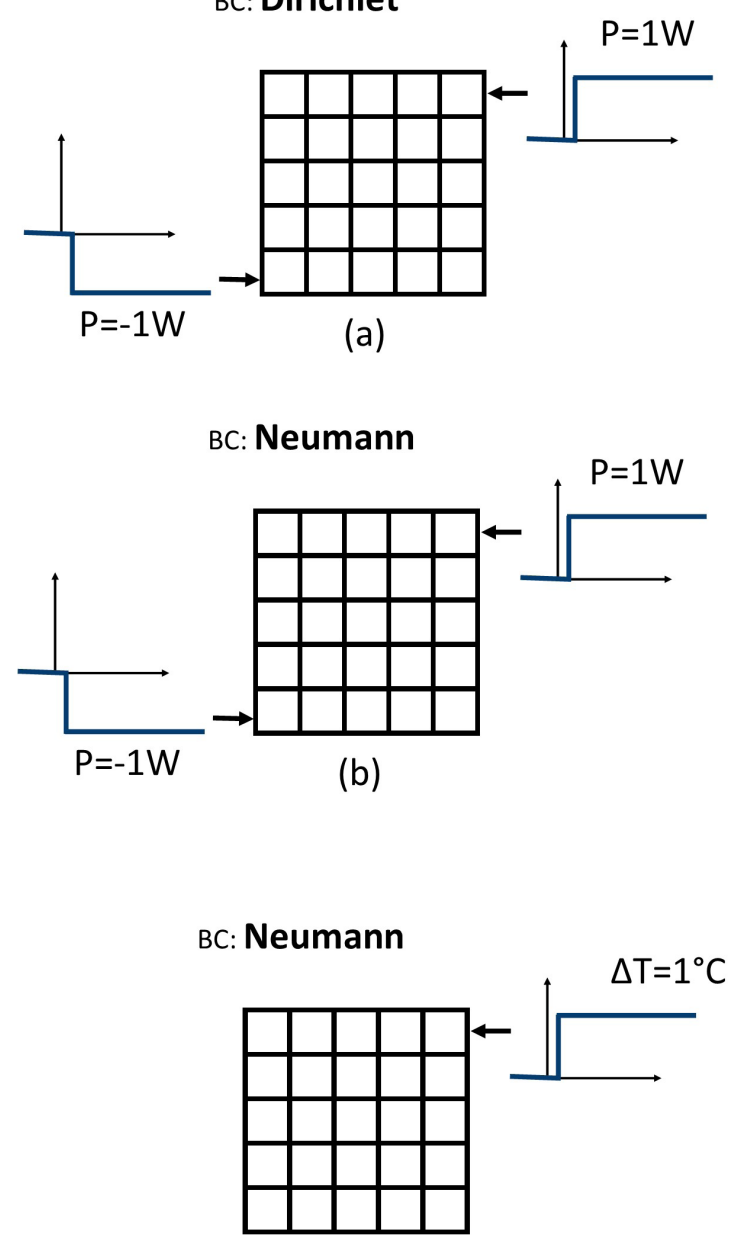

(c)

Fig. 5 Boundary conditions for different simulations 


\section{Results}

The difference between the results of the conventional time-domain integration scheme (the widely known, simple and stable reverse Euler method) was implemented and the results obtained by our new, transfer function based calculation method is investigated by using the above described simple test case (Fig. 5). The applied thermal loads (excitations) were the following:

- forced step-wise heat-flux,

- forced, step-wise temperature rise.

The results obtained by our new calculation method are theoretically accurate as they are analytically calculated; these results are free of the inherent errors of the approximate numerical solution methods such as the numerical timedomain integration scheme. Therefore, these transfer function calculation based results were considered as baseline when we compared them with the results obtained by a conventional simulation and with the results obtained by our new reduced order modeling technique. In case of transient simulation of the conventional solver based on the reverse Euler method, the inherent numerical error is proportional with the applied $\Delta t$ simulation time step, thus, in order to keep this inherent error small the simulation time-step has to be kept sufficiently small. (It has to be smaller than half of the smallest time-constant that we want to account accurately for.)

\subsection{Basic error analysis}

After the node reduction process only two terminal nodes remained which were originally connected to the two transverse corners of the simulated rectangular area (see Fig. 5). The boundary condition settings were the following:

- Dirichlet-type conditions with $0^{\circ} \mathrm{C}$ applied at the eliminated nodes

- and Neumann-type conditions for the monitoring nodes with $\pm 1 \mathrm{~W}$ heat flux,

as it is shown in Fig. 5. The transient simulation results with different time-steps are shown in Fig. 6. The error can be calculated as the average of the absolute value of the relative difference:

Error $=\sum_{t_{i}} \frac{T_{T f}\left(t_{i}\right)-T_{n u m}\left(t_{i}\right)}{T_{T f}\left(t_{i}\right)}$

where $T_{T f}$ denotes the temperature response obtained by our new, analytical transfer function based method

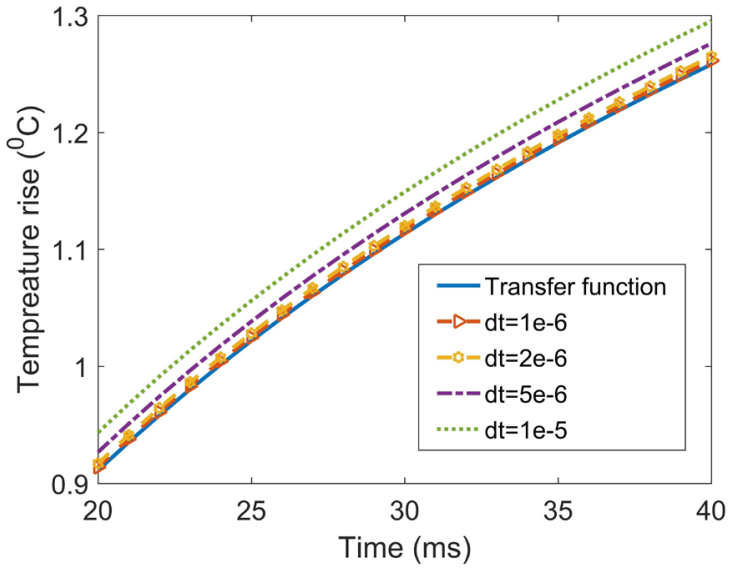

Fig. 6 The effect of changing the time-step

\begin{tabular}{lc} 
Table 1 Effect of the size of time-step on the error \\
\hline Time-step (s) & Error $\left(\times 10^{-5}\right)$ \\
\hline $10^{-5}$ & 930 \\
$5 \cdot 10^{-6}$ & 460 \\
$2 \cdot 10^{-6}$ & 180 \\
$10^{-6}$ & 89 \\
$10^{-7}$ & 7.9 \\
$10^{-8}$ & 0.9 \\
$10^{-9}$ & 0.09 \\
$10^{-10}$ & 0.009
\end{tabular}

and $T_{\text {num }}$ is the temperature response calculated by the numerical time-domain integration scheme. The results are summarized in Table 1. As it is visible the error is linearly decreasing with the size of the $\Delta t$ time-step corresponding to the known feature of the Euler-type time-domain integration scheme.

In the second test run the same structure was simulated as before, but with $0 \mathrm{~W}$ Neumann-type boundary condition as shown in Fig. 5 (b). The result are presented in Fig. 7. The size of the time-step of the time domain integration of the classical SUNRED solver was $10^{-7} \mathrm{~s}$.

In the third test run with the same test structure, temperature step excitation was applied at one of the terminals, and the temperature change on the other terminal was recorded as a response to this - see Fig. 5 (c). The obtained results are shown in Fig. 8.

\section{LED package model implementation and testing 7.1 Simulation setups}

The test setup for a LED model is a simplified structure of a packaged LED (with dimensions typical for today's midpower/high-power LEDs) assembled to a Metal Core Printed 
Circuit Board (MCPCB) and cooled with a heat-sink. The dimensions and the structure of the modelled LED assembly is shown in Fig. 9 and are detailed in Table 2.

In the assumed structure the LED-chip is mounted to an aluminium-nitride ceramic board, which is soldered to an MCPCB substrate. The MCPCB is attached to a heat sink with a thin $(75 \mu \mathrm{m})$ layer of thermal interface material denoted as TIM (often also referred to as TIM2 layer).

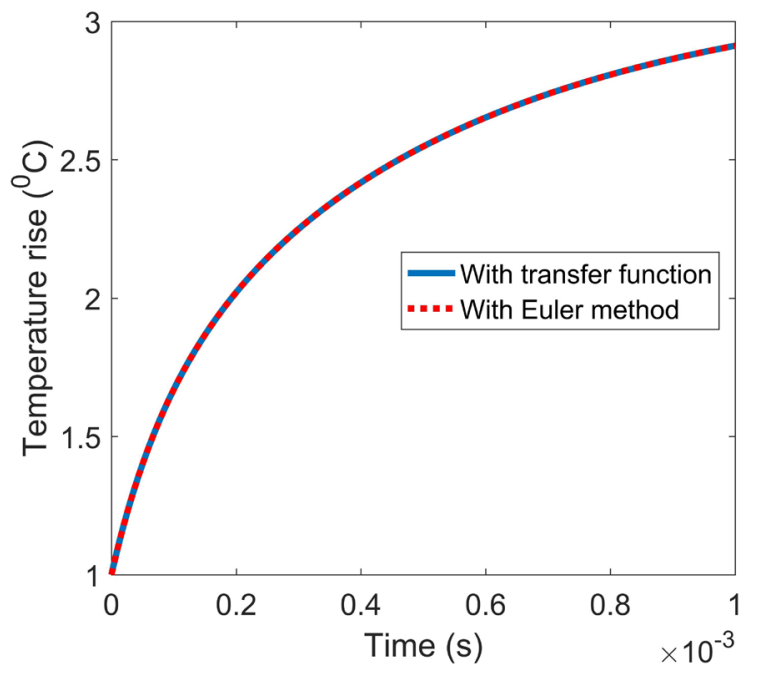

Fig. 7 Results for Neumann boundary condition (b)

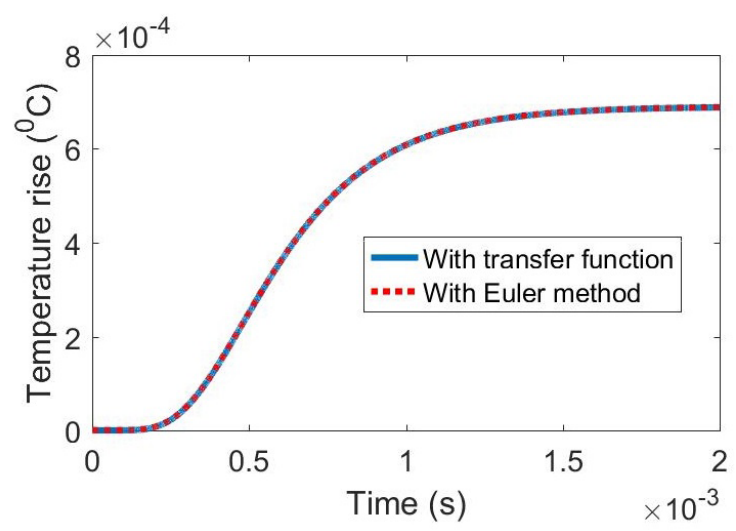

Fig. 8 Results for isothermal boundary condition (c)

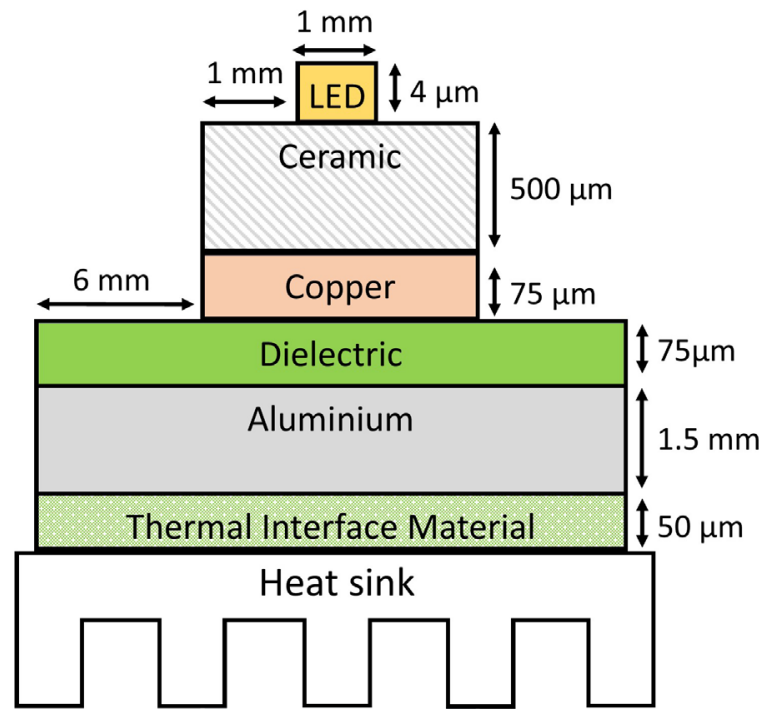

Fig. 9 The assumed geometry of the LED package

In the assumed structure the LED-chip is mounted to an aluminium-nitride ceramic board, which is soldered to an MCPCB substrate. The MCPCB is attached to a heat sink with a thin $(75 \mu \mathrm{m})$ layer of thermal interface material denoted as TIM (often also referred to as TIM2 layer).

The spatial resolution of the mesh in the SUNRED model is varying in the different layers of the structure, matched to the layer thicknesses and the lateral dimensions of the structural elements in question. The modelled geometry contained 216 building blocks, and 1080 nodes. The heterogeneous material distribution was handled in a non-parametric way (constant material property values were assumed for all simulations), except the TIM layer (representing the interface between the heat sink and the aluminium bulk of the MCPCB) where the $\lambda$ thermal conductivity of the TIM was a parameter varied during some of our simulations.

The boundary conditions of the model are shown in Fig. 10. The sides of the structure were assumed to be adiabatic (with zero heat-flux across) while at the bottom isothermal

Table 2 Physical dimensions of the structure and the properties of the considered materials

\begin{tabular}{lccccc}
\hline Layer & Thickness $(\mu \mathrm{m})$ & Size $(\mathrm{mm})$ & Resolution $(\mu \mathrm{m})$ & Thermal conductivity $\left(\mathrm{W} / \mathrm{m}^{2} \mathrm{~K}\right)$ & Specific Heat $\left(\mathrm{kJ} / \mathrm{m}^{3}\right)$ \\
\hline LED-chip & 4 & 1 & & 130 & 3014 \\
AlN ceramic & 500 & 3 & $250 \times 250$ & 240 & 301 \\
Copper & 75 & 3 & $250 \times 75$ & 2.2 & 24 \\
2457Dielectric & 75 & 15 & $750 \times 750$ & 787 \\
Aluminium & 1500 & 15 & $750 \times 75$ & 2457 \\
TIM & 75 & 15 & & 1600 \\
\hline
\end{tabular}




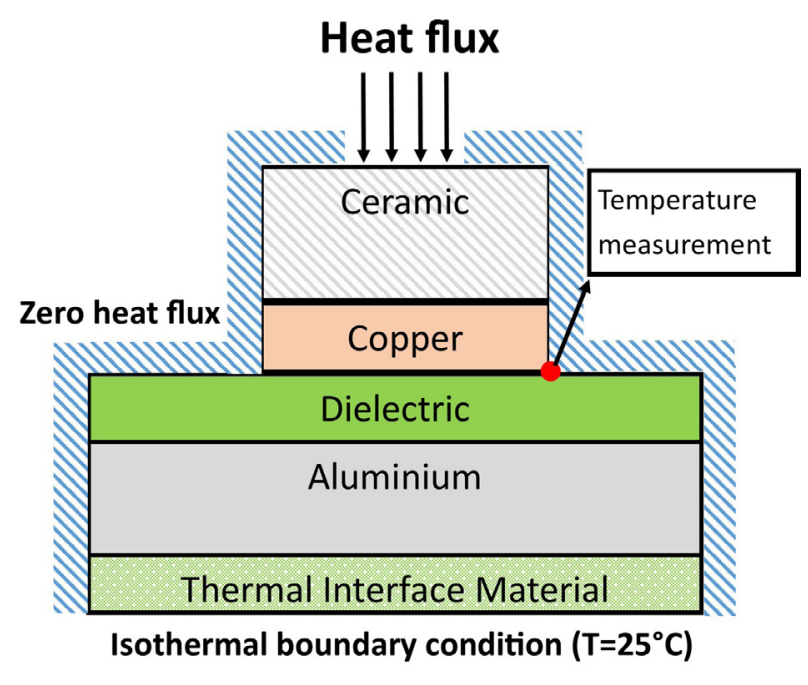

Fig. 10 The boundary conditions and monitoring points of the LED package

conditions with a fixed, constant $25^{\circ} \mathrm{C}$ temperature. The monitoring nodes are the upper nodes of the LED-chip, where we assumed uniform heat generation (the "junction") and a single node on the MCPCB (the aluminium layer) which is assumed as a temperature measuring point (also known as thermal test point). This test point was located 2 $\mathrm{mm}$ from the edge of the MCPCB.

In the first test run we aimed to determine the temperature of the monitoring points (junction node, thermal test point) assuming the following power dissipation patterns at the junction:

a) Step-wise change of the dissipated power at the junction (with $1 \mathrm{~W}$ height) for different values of the $\lambda$ parameter,

b) Periodic heating power typical for directly $\mathrm{AC}$ voltage driven LEDs.

With the first case we can investigate how the TIM thermal conductivity influences the relation of the test point temperature and the junction temperature (transient signal delay, steady-state temperature attenuation). The second example would tell us what would be the actual temperature waveforms of directly AC driven LEDs and how the mean value of the junction temperature and/or the test point temperature would change.

There is a strong practical interest in creating compact thermal models of thermal transfer impedance(s) between the LED junction and the thermal test point(s). The reason is that under application conditions of LEDs it is difficult to monitor the absolute value of the LED junction temperature but with a thermistor or a thermocouple attached to the thermal test point of the MCPCB one can obtain temperature information about the system. This is importante.g. for overheating protection of a LED luminaire or it can also be used as input for an embedded multidomain LED model used for temperature compensating dimming schemes in order to maintain the so called isoflux operation of LED streetlighting luminaires, see for example [26, 27]. The question is of course how relevant is the temperature information obtained at the thermal test point when one wants to draw conclusions regarding the junction node, i.e. what information about the thermal excitation applied at the junction remains available in the temperature waveforms measured at the test point?

Obtaining the heat dissipation (and/or the junction temperature) from the temperature signal of the thermal monitoring is an inverse problem which is not as straightforward to solve as obtaining the temperature response of any point in the system when the dissipation (the excitation) is known.

The thermal system we investigate is a causal system characterized by a $H(s)$ transfer function in the complex frequency (Laplace) domain. With a homogeneous heat flux $\left(I_{T h, L E D}\right)$ excitation at the junction we can write:

$T_{M P}(s)=H(s) I_{T h, L E D}(s)$

where $H(s)=\underline{\underline{Y}}_{M P, L E D}(s)$ describes a causal system, i.e. there are more poles than zeros in the $H(s)$ transfer function. The above outlined problem is formulated as:

$$
I_{T h, L E D}(s)=\frac{1}{H(s)} T_{M P}(s)
$$

where $1 / H(s)$ describes a non-causal system, containing more zeros than poles on the complex plain (s-domain).

For continuous signals there is no problem with the backward transformation to obtain the $I_{T h, L E D}(s)$ excitation function, but for a discrete system (such as our numerical simulation model) there is no simple method to perform this. For discretized systems, there exist other integraltransformation methods, for example the Z-transform, or Fourier-transform. If we chose the Fourier-transform as it can be easily implemented in a numerical simulation environment with the help of the FFT algorithm, the heat flux can be calculated as:

$$
I_{T h, L E D}(\omega)=\frac{1}{H(j \omega)} T_{M P}(\omega)
$$


The temperature of the LED-chip can be calculated as:

$$
T_{L E D}=H_{2}(j \omega) \frac{1}{H(j \omega)} T_{M P}(\omega)
$$

where $H_{2}(s)=\underline{\underline{Y}}_{L E D, M P}^{-1}(s)$. Note, that the operation defined by Eq. (21) is also called deconvolution.

The testing method starts with the estimation of the $T_{i}$ "measured" temperature with the sampling frequency $f_{s}$. The $T_{M P}(\omega)$ sequence comes from the FFT transform of $T_{i}$, the values of $\omega$ are taken equidistantly from the $\left[0 . .2 \pi f_{s}\right]$ interval. After the element-by-element multiplication with $1 / H(j \omega)$ the resulting series of samples of the $\left(I_{T h, L E D}(\omega)\right)$ heat flux can be inverse transformed in order to obtain the $I_{T h, L E D}(t)$ sequence of time-domain samples.

Under realistic conditions, of course, a low-pass filter should also be applied to the frequency-domain samples. Note, that with this method signals with smooth, slow transitions can be deconvolved only; in case of rapidly changing signals it is worthwhile to carry out the deconvolution of measured data based on the $R(t)$ pulse response (i.e. the weight function) [28]. For details on limits of application of deconvolution based methods to such thermal problems refer also to the fundamental paper of V. Székely [28].

In a case such as our LED example the heat-flux (as excitation) is determined as follows:

$$
I_{T h, L E D}\left(t_{i}\right)=T_{M P}\left(t_{i}-d t\right) / R(d t),
$$

where the $R$ is the pulse response function with a given resolution. The remaining part of the pulse response function is subtracted from temperature:

$$
T_{M P}(\tau)_{\text {new }}=T_{M P}(\tau)_{\text {old }}-R(\tau) \text {, }
$$

where $\tau$ represents the time instances beyond $t_{i}$. In principle this approach can be used to perform the deconvolution but since the value of $R(d t)$ is typically very small, any noise (including the numerical errors due to the finite resolution of the computer representation of the real numbers) results in divergence of the numerical deconvolution. As practical workaround to this problem, to achieve stable results, a few $(k)$ initial samples of the pulse response should be discarded, thus Eq. (23) is to be modified as follows:

$$
I_{T h, L E D}\left(t_{i}-k \cdot d t\right)=T_{M P}\left(t_{i}\right) / R(k \cdot d t)
$$

The relative error originating from this approximation can be defined as the ratio of the sum of the first $k-1$ samples and the $k^{\text {th }}$ sample of the pulse response function. As a test case, we can choose any of the analytically obtained step response functions (shown in Fig. 11), or their

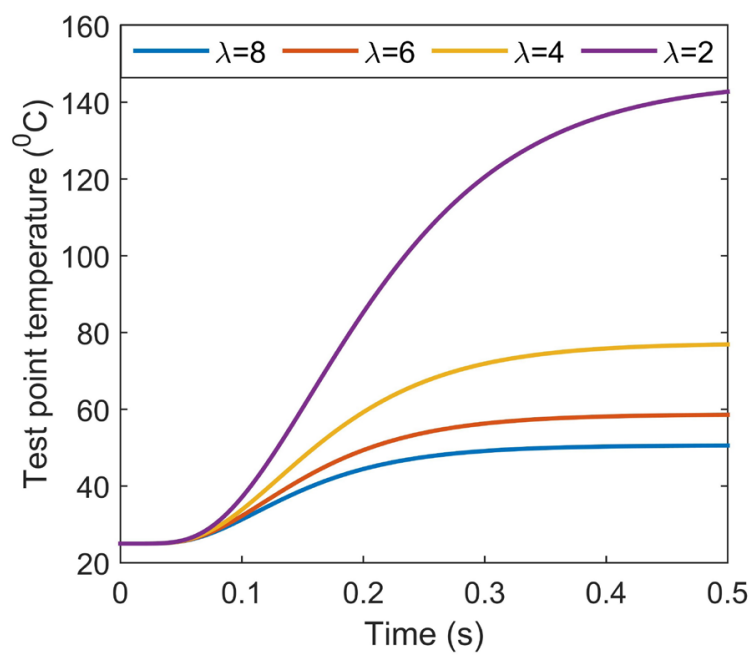

Fig. 11 The temperature values on the thermal test point with different TIM conductivity $(\lambda=2,4,6,8 \mathrm{~W} / \mathrm{mK})$

linear combination (e.g. square wave). The pure, original form of the $I_{T h, L E D}(t)$ is known [unit step function or square function]. The result of the solution of the inverse problem can be compared to this. After solving the inverse problem, the obtained $I_{T h, L E D}(t)$ function was applied as stimulus in a traditional transient simulation. The method can be benchmarked by the difference of the original $T_{i}$ values and corresponding $T_{i}$ values of the samples of the test point temperature obtained by a classical transient simulation.

\section{Results}

The first simulations setup (a) (shown in Figs. 9 and 10) was investigated by a parametric analysis. The parameter we varied was the $\lambda$ thermal conductivity of the TIM 2 layer as indicated in Table 2. In the first place we were interested in the transient temperature response at the thermal test point assuming different $\lambda$ parameters. The simulated results are shown in Fig. 20. The steady-state temperature of the test point as function of the $\lambda$ parameter is shown in Fig. 12.

We found that (according to the prior expectations) the steady-state temperatures highly depend on the thermal conductivity of the TIM2 material. This is mainly because the lateral heat spreading in the MCPCB substrate is getting more pronounced with decreasing thermal conductivity of the TIM2 layer.

For the second test case (b) we used real, measured heat dissipation data of a directly AC-driven LED package, which is borrowed from [29]. The waveform of the heat dissipation of such an LED is shown in Fig. 13 The heating power of the LED is a periodic signal with 


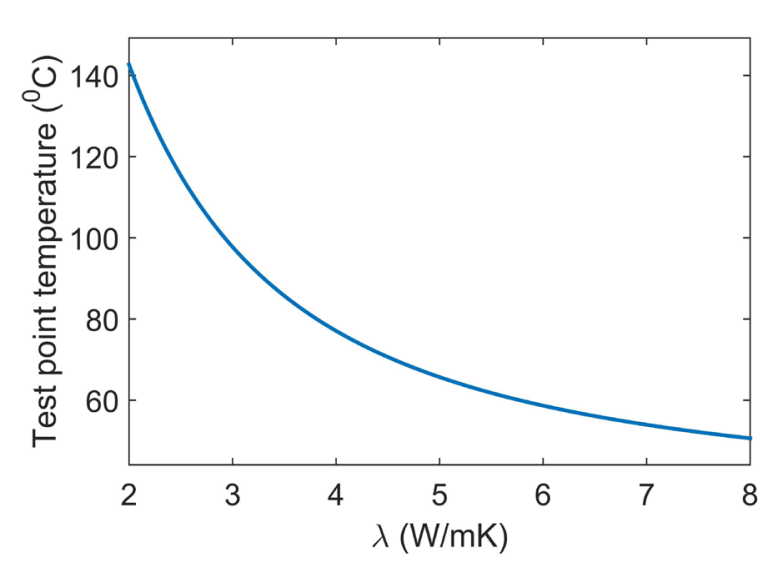

Fig. 12 The steady-state temperature values on the measurement point with different TIM conductivity $(\lambda=2,4,6,8 \mathrm{~W} / \mathrm{mK})$

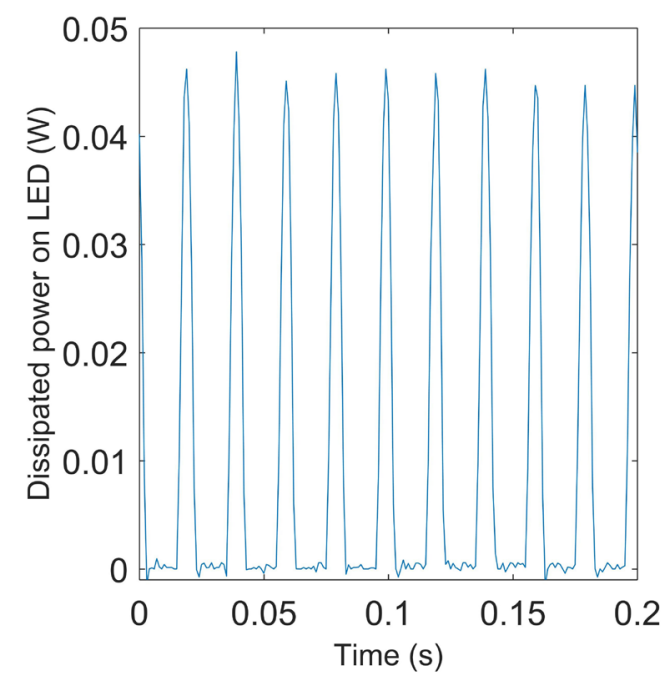

Fig. 13 Periodic heating power of a directly AC-voltage driven LED chip [29]

multiple significant harmonics up to $300 \mathrm{~Hz}$ of the $50 \mathrm{~Hz}$ base frequency with an amplitude of $\sim 0.5 \mathrm{~W}$. The duration of the non-zero part of the heating power waveform $8 \mathrm{~ms}$ width. The average power was $0.1 \mathrm{~W}$. In principle a periodic heating results also in a periodic temperature response but the temperature signal at the thermal test point of the MCPCB is a smooth function (due to the low-pass filter nature of thermal "transmission line" between the driving point and the monitoring point) as illustrated in Fig. 14. There is only a $6^{\circ} \mathrm{C}$ temperature rise on the measurement point, but the LED junction temperature shown in Fig. 15 follows the periodicity of the heating power. Having lost all the details of fast changes in the signal of the thermal test point means, that there are fundamental limits for solving the inverse problem, see [28] for further details.

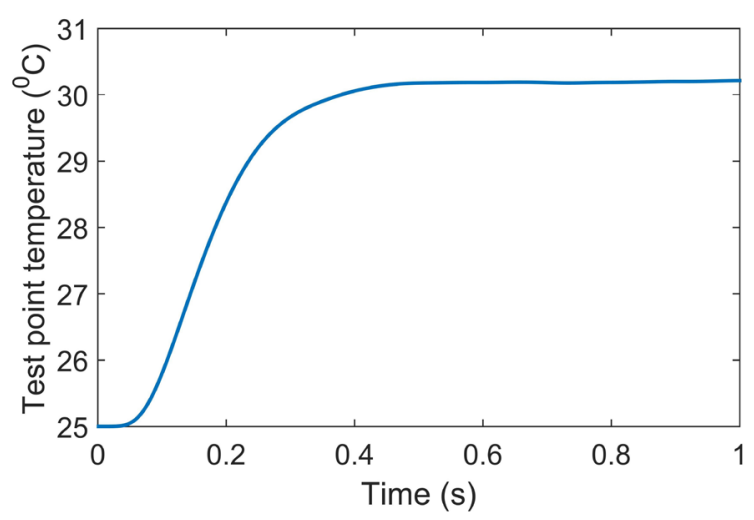

Fig. 14 Temperature of the thermal test point as response to the periodic heating power with the waveform shown in Fig. $13(\lambda=4 \mathrm{~W} / \mathrm{mK})$

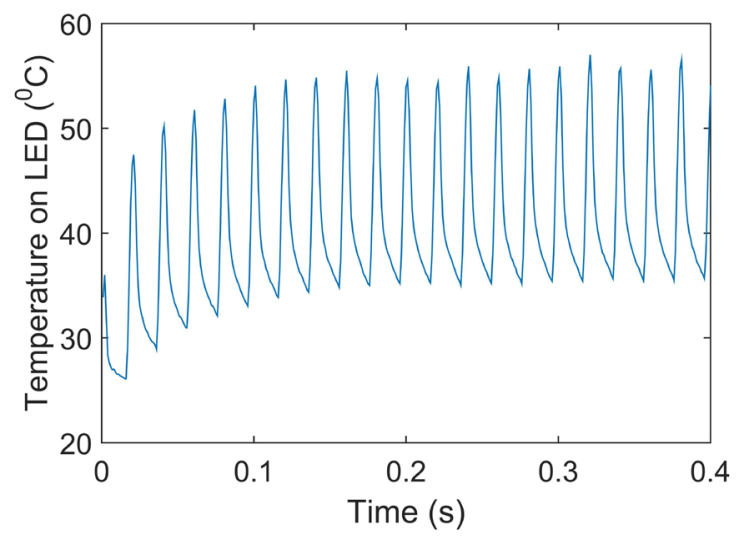

Fig. 15 Temperature of the LED surface (junction) as response to the periodic heating power with the waveform shown in Fig. 13

$$
(\lambda=4 \mathrm{~W} / \mathrm{mK})
$$

As an example to demonstrate the solution of an inverse problem we assumed a square wave shaped heating power function at the LED junction with a $50 \%$ duty cycle, a 1 $\mathrm{W}$ amplitude and $400 \mu$ s period. The temperature values of the thermal test point as response to such an excitation are shown in Fig. 16. For the sake of a realistic simulation, we added noise to these obtained test point temperature values, assuming different $A$ amplitude values.

We investigated the effect of the amplitude of the noise on the results of the deconvolution. The input power was calculated back from the sampled noisy temperature data according to Eq. (24). The sampling frequency was $1 \mathrm{kHz}$. The heat flux at the LED junction calculated back for different noise amplitudes is shown in Fig. 17. The "restored" dissipation signal was used in a second, conventional transient simulation in order to obtain back the temperature function at the thermal test point again. The original "measured" and this re-simulated temperature pulses are shown in Fig. 18. 


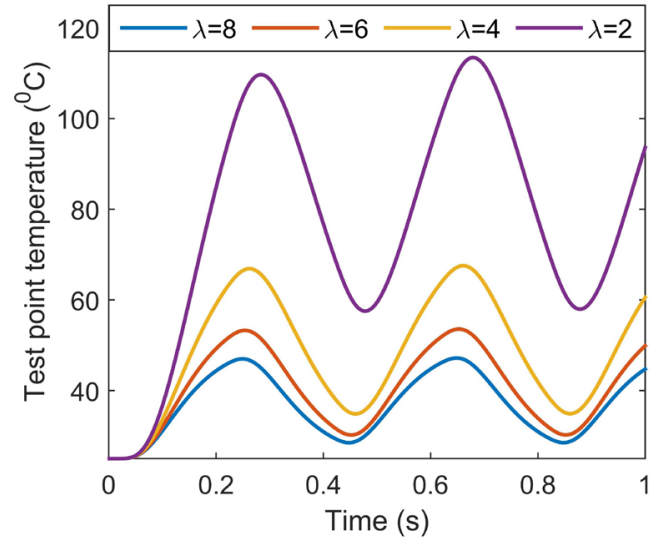

Fig. 16 The temperature of the thermal test point obtained as a response to a square wave-shaped heating at the LED's junction, simulated with the parameter values $\lambda=2,4,6,8 \mathrm{~W} / \mathrm{mK}$

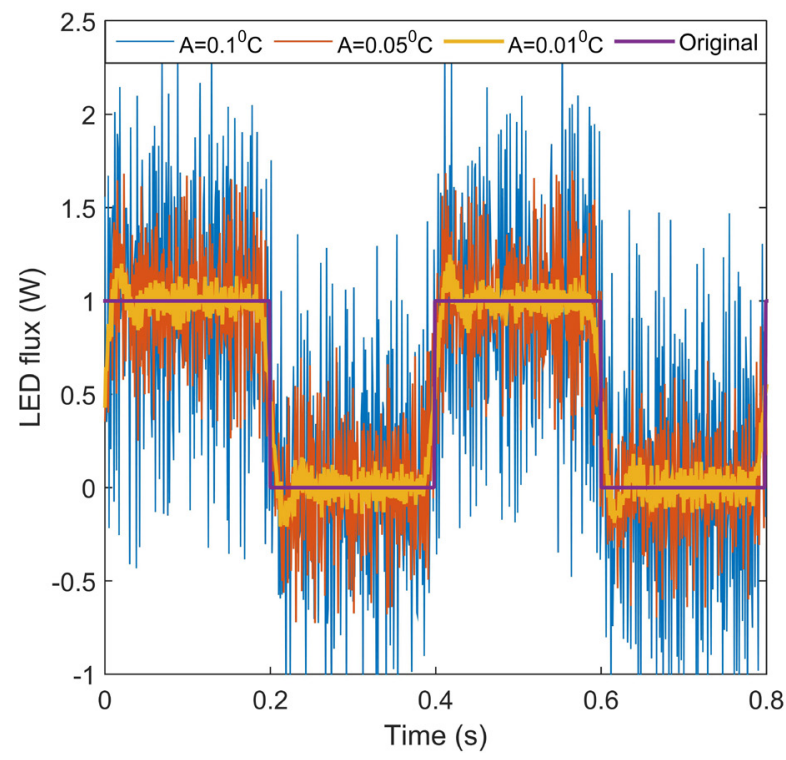

Fig. 17 The heat flux waveform obtained as a result of the solution of the inverse problem, assuming different noise amplitudes $(\lambda=4 \mathrm{~W} / \mathrm{mK})$

We defined the error of the method as the variance between the original temperature signal of the measurement point and temperature data obtained by the second conventional transient simulation:

$$
\sigma^{2}=\frac{\sum\left(T_{\text {original }}(i)-T_{\text {reconvolved }}(i)\right)^{2}}{N},
$$

where $N$ is the number of sampling points (1000). The relative error can be calculated as the ratio of the variance and the average temperature rise.

$$
\text { Error }=\frac{\sigma}{\sum\left(T_{\text {original }}(i)-25\right) / N}
$$

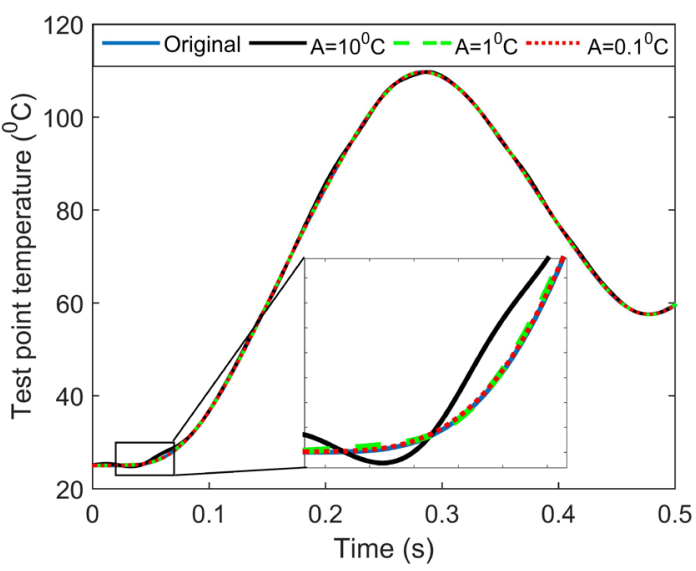

Fig. 18 Comparison of the temperature signals at the thermal test point obtained for a known heating power waveform and for a heating power waveform obtained as the result of solving the inverse problem (for parameter value $\lambda=4 \mathrm{~W} / \mathrm{mK}$ ) with different noise amplitude values

The relative errors calculated for different noise levels (different $A$ amplitudes), are shown in Fig. 19.

\subsection{Results with balanced model reduction}

The greatest disadvantage of the presented method is its huge execution time. To overcome this problem, we propose to use the order reduction algorithm in the complex frequency domain in each step of building the model: in each connection step and after each node-reduction step. The detailed description of the balanced order reduction algorithm can be found in the literature [30]. According to this, the balanced order reduction is performed only on low order systems (with $N$ less than 20), therefore the computational cost is still kept low.

For testing the balanced model order reduction, we moved the thermal test point closer to the LED chip in order to see the difference between the results of the reduced order model and the analytical results obtained for the un-reduced model. First, we investigated the system with the step function excitation (case LED (a)). The parameter varied was the number of dominant Hankel values to which the state-space was projected. In the first place we were interested in the transient temperature response at the thermal test point. The simulated results are shown in Fig. 20.

We found that (according to the prior expectations) the error of the reduced order simulation is highly influenced by the number of dominant values, however the results do not change significantly with the order above 6 .

For the second case (b) we used the real periodic heat dissipation shown in Fig. 13. Due to the low-pass filter 


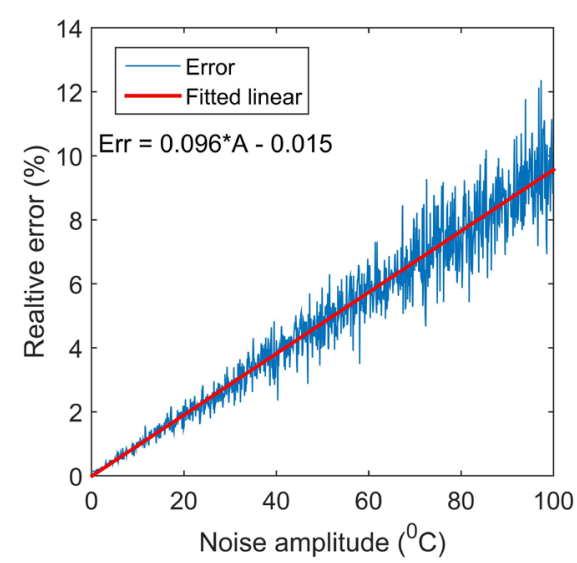

Fig. 19 The relative error as function of the noise amplitudes $(\lambda=4 \mathrm{~W} / \mathrm{mK})$

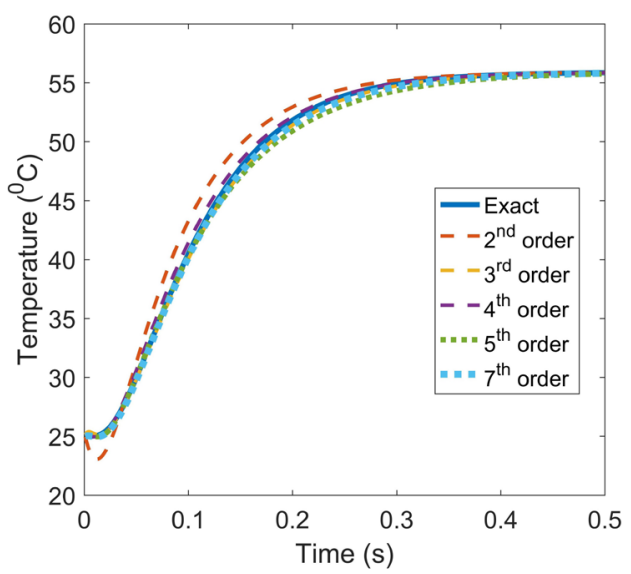

Fig. 20 The temperature values on the thermal test point with different orders used in the approximation of the transfer function $(2,3,4,5$ and 7$)$

nature of the thermal transfer impedances the temperature signal at the thermal test point usually should be a smooth function which, due to the short distance, thus, a stronger coupling between the driving point and the monitoring point is not the case here, see Fig. 21.

We defined the error of the method as the variance between the original temperature signal of the measurement point and temperature data obtained by the second conventional transient simulation:

$\sigma^{2}=\frac{\sum\left(T_{\text {original }}(i)-T_{\text {Re duced }}(i)\right)^{2}}{N_{S p}}$,

where $N_{S p}$ is the number of sampling points (1000). The relative error can be calculated as the ratio of the variance and the average temperature rise.

$$
\text { Error }=\frac{\sigma}{\sum\left(T_{\text {original }}(i)-25\right) / N S p}
$$

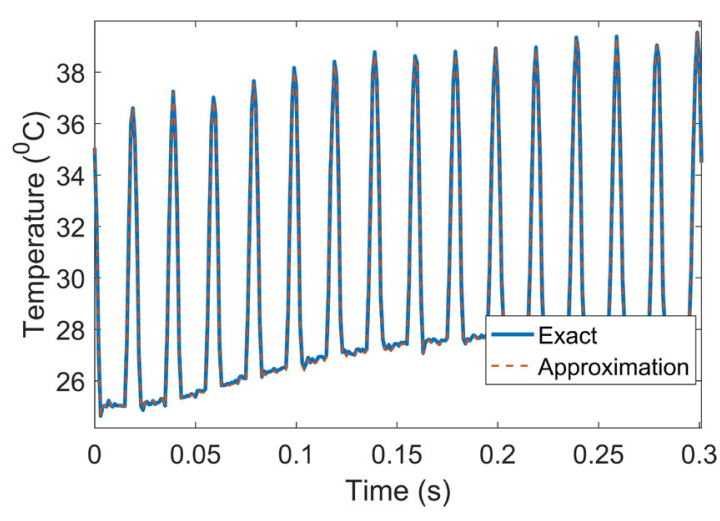

Fig. 21 Temperature of the thermal test point as response to the periodic heating power with the waveform shown in Fig. 13 $4^{\text {th }}$ order approximation

The relative errors calculated for different order of approximation levels (different numbers of Hankel values to keep), are detailed in Table 3.

\subsection{Computational complexity}

While the model is being built, only the surface nodes behave as monitoring points, therefore the size of the admittance matrix is proportional to the number of nodes on the surface. In 2D we assume that the computational cost is proportional to $N^{2}$, where $N$ is the number of monitoring nodes in the current state of reduction. The number of surficial nodes (these are the monitoring nodes during the reduction process) is proportional to $N$, and the cost of the matrix operations are proportional to $N^{2}$. For the sake of simplicity we examined the former rectangular structure but with more nodes inside. The measured runtimes using balanced order reduction of the transfer functions to the $6^{\text {th }}$ order are summarized in Table 4. These runtimes are the worst-case results, because of the used square shaped field. The implementation was done by Matlab 2016b, with the help of the built-in balred () function. The runtimes shows that the computational cost increasing near linearly, which is a consequence of the 2D modeling. This indicates that the new method is highly effective on flat structures, such as LEDs.

Table 3 Calculated error and reduction time for the results presented in Fig. 21

\begin{tabular}{lccc}
\hline Order & Error (a) (\%) & Error (b) (\%) & Reduction time (s) \\
\hline 2 & 3.5 & 3.8 & 5 \\
3 & 0.7 & 0.6 & 6 \\
4 & 1.3 & 0.4 & 7 \\
5 & 1.0 & 0.9 & 7 \\
7 & 0.6 & 0.5 & 8 \\
\hline
\end{tabular}


Table 4 Runtime versus number of nodes (DoF) with balanced order

\begin{tabular}{rr}
\multicolumn{2}{r}{ reduction of a 2D square shaped structure } \\
\hline Number of nodes $(n)$ & Runtime (s) \\
\hline 320 & 1.3 \\
1280 & 1.6 \\
5120 & 2.1 \\
20480 & 3.5 \\
81920 & 8.5 \\
327680 & 28 \\
1310720 & 103 \\
5242880 & 258 \\
20971520 & \\
\hline
\end{tabular}

\section{Conclusions}

We proposed a new modeling method that can be considered as a reduced order or compact modeling technique for the efficient handling of thermal transfer impedances. The new modeling method is accurate, parametric and boundary condition independent. For three different simple test cases the results obtained by the proposed method were compared to the results obtained by a conventional algorithm based thermal simulation code. The reduction scheme is naturally time-consuming, in our simple test case it took 14 seconds.

The basic idea of the combination of SUNRED algorithm with transfer function was completed with a reduction method in the complex frequency plane. The results shows that the balanced truncation is suitable for the process. The runtimes were dropped to its $0.5 \%$, even though this reduction method is based on single value decomposition, which is traditionally slow (compared with Krylov subspace methods).

\section{References}

[1] Lasance, C. J. M. "Ten Years of Boundary-Condition-Independent Compact Thermal Modeling of Electronic Parts: A Review", Heat Transfer Engineering, 29, pp. 149-168, 2008. https://doi.org/10.1080/01457630701673188

[2] Methodology for the Thermal Modeling of Component Packages, JEDEC JESD15 Standard, 2008.

[3] DELPHI Compact Thermal Model Guideline, JEDEC JESD15 Standard, 2008.

[4] DELPHI Compact Model Guideline - JEDEC JC 15.1 Committee proposed document (JESD 15-4), JEDEC JESD15 Standard, 2008.

[5] Blackmore, B. "Automatic calibration of detailed IC package models", In: 2016 32nd Thermal Measurement, Modeling \& Management Symposium (SEMI-THERM), San Jose, CA, USA, March 14-17, 2016. pp. 105-112.

https://doi.org/10.1109/SEMI-THERM.2016.7458454
The reduced order model of the thermal transfer impedances can be used as an analytic model in a frequency-domain deconvolution scheme to solve certain kinds of inverse problems, e.g. to try to restore the dissipation waveform from the temperature signal measured at the thermal test point of an LED assembly. We presented a practical method to realize such a deconvolution and we highlighted some limits of such a waveform reconstruction. We defined an error metric for the proposed inverse problem solution method.

One of our test cases was a simplified structure of an MCPCB assembled packed LED chip. The model reduction for a parametric simulation example took about 23 minutes but for non-parametric studies it took only 3 minutes. The obtained reduced order model of thermal transfer impedances proved to be very useful in some parametric studies. With this example we also demonstrated how thermal behavior at the LED junction can be predicted from temperature data measured at the thermal test point of the LED assembly.

\section{Acknowledgement}

The contribution of the European Union for supporting this study in the context of the H2020 ECSEL Joint Undertaking program (2016-2019) within the frames of the Delphi4LED project (grant agreement 692465) is acknowledged. Co-financing of the Delphi4LED project by the Hungarian government through the NEMZ_16-12017-0002grant of the National Research, Development and Innovation Fund is also acknowledged.

[6] Poppe, A., Zhang, Y., Wilson, J., Farkas, G., Szabo, P., Parry, J., Rencz, M., Szekely, V. "Thermal Measurement and Modeling of Multi-Die Packages", IEEE Transactions on Components and Packaging Technologies, 32(2), pp. 484-492, 2009. https://oi.org/10.1109/TCAPT.2008.2004578

[7] Lasance, C. J. M. "The European project PROFIT: prediction of temperature gradients influencing the quality of electronic products", In: Seventeenth Annual IEEE Semiconductor Thermal Measurement and Management Symposium (Cat. No.01CH37189), San Jose, CA, USA, March 22, 2001, pp. 120-125. https://doi.org/10.1109/STHERM.2001.915160

[8] Schweitzer, D., Pape, H. "Boundary condition independent dynamic thermal compact models of IC packages", In: 2003 9th International Workshop on Thermal Investigations of ICs and Systems (THERMINIC), Aix-en-Provence, France, Sept. 24-26, 2003, pp. $225-230$. 
[9] Schweitzer, D. "Generation of multisource dynamic compact thermal models by RC-network optimization", In: 29th IEEE Semiconductor Thermal Measurement and Management Symposium (SEMITHERM), San Jose, CA, USA, March 17-21, 2013, pp. 116-123. https://doi.org/10.1109/SEMI-THERM.2013.6526815

[10] Schweitzer, D., Ender, F., Hantos, G., Szabó, P. G. "Thermal transient characterization of semiconductor devices with multiple heat sources-Fundamentals for a new thermal standard", Microelectronics Journal, 46(2), pp. 174-182, 2015. https://doi.org/10.1016/j.mejo.2014.11.001

[11] Székely, V., Poppe, A., Rencz, M., Csendes, A., Páhi, A. "Electrothermal simulation: a realization by simultaneous iteration", Microelectronics Journal, 28(3), pp. 247-262, 1997. https://doi.org/10.1016/S0026-2692(96)00029-8

[12] Poppe, A., Hegedus, J., Szalai, A., Bornoff, R., Dyson, J. "Creating multi-port thermal network models of LED luminaires for application in system level multi-domain simulation using spicelike solvers", In: 2016 32nd Thermal Measurement, Modeling \& Management Symposium (SEMI-THERM), San Jose, CA, USA, March 14-17, 2016, pp. 44-49. https://doi.org/10.1109/SEMI-THERM.2016.7458444

[13] Poppe, A. "Simulation of LED Based Luminaires by Using MultiDomain Compact Models of LEDs and Compact Thermal Models of their Thermal Environment", Microelectronics Reliability, 72, pp. $65-74,2017$. https://doi.org/10.1016/j.microrel.2017.03.039

[14] Codecasa, L. "Nonlinear dynamic compact thermal models by structurepreserving projection", Microelectronics Journal, 45(12), pp. 1764-1769, 2014. https://doi.org/10.1016/j.mejo.2014.06.001

[15] Codecasa, L., d' Alessandro, V., Magnani, A., Rinaldi, N. "Structure-preserving approach to multi-port dynamic compact models of nonlinear heat conduction", Microelectronics Journal, 46(12), pp. 1129-1137, 2015. https://doi.org/10.1016/j.mejo.2015.09.021

[16] Codecasa, L., d' Alessandro, V., Magnani, A., Rinaldi, N., Zampardi, P. J. "Fast novel thermal analysis simulation tool for integrated circuits (FANTASTIC)", In: 20th International Workshop on Thermal Investigations of ICs and Systems (THERMINIC), London, UK, Sept. 24-26, 2014, pp. 1-6. https://doi.org/10.1109/THERMINIC.2014.6972507

[17] Codecasa, L., d' Alessandro, V., Magnani, A., Rinaldi, N. "Calibration of detailed thermal models by parametric dynamic compact thermal models", In: 2016 22nd International Workshop on Thermal Investigations of ICs and Systems (THERMINIC), Budapest, Sept. 21-23, 2016, pp. 69-75. https://doi.org/10.1109/THERMINIC.2016.7748650

[18] Codecasa, L. "Compact Models of Dynamic Thermal Networks with Many Heat Sources", IEEE Transactions on Components and Packaging Technologies, 30(4), pp. 653-659, 2007. https://doi.org/10.1109/TCAPT.2007.906316

[19] Antoulas, A. C., Beattie, C. A., Gugercin, S. "Interpolatory model reduction of large-scale dynamical systems", In: Mohammadpour, J., Grigoriadis, K. (eds.) Efficient Modeling and Control of LargeScale Systems, Springer, Boston, MA, 2010, pp. 3-58. https://doi.org/10.1007/978-1-4419-5757-3_1
[20] Kohári, Z., Székely, V., Rencz, M., Páhl, A., Dudek, V., Höfflinger, B. "Studies on the heat removal features of stacked SOI structures with a dedicated field solver program (SUNRED)", Microelectronics Reliability, 38(12), pp. 1881-1891, 1998. https://doi.org/10.1016/S0026-2714(98)00199-1

[21] Páhi, A., Székely, V., Rosenthal, M., Rencz, M. "3D extension of the SUNRED field solver", In: 1998 4th International Workshop on Thermal Investigations of ICs and Systems (THERMINIC), Cannes, France, Sept. 27-29, 1998, pp. 185-190.

[22] Pohl, L. "Multithreading and Strassen's algorithms in SUNRED field solver", In: 2008 14th International Workshop on Thermal Inveatigation of ICs and Systems (THERMINIC), Rome, Sept. 24-26, 2008, pp. 137-141.

https://doi.org/10.1109/THERMINIC.2008.4669895

[23] Nemeth, M., Jani, L., Poppe, A. "Compact modeling approach for microchannel cooling aimed at high-level thermal analysis of 3D packaged ICs", In: 2016 Symposium on Design, Test, Integration and Packaging of MEMS/MOEMS (DTIP), Budapest, 30 May - 2 June, 2016, pp. 1-6. https://doi.org/10.1109/DTIP.2016.7514865

[24] Treurniet, T., Lammens, V. "Thermal management in color variable multi-chip LED modules", In: Twenty-Second Annual IEEE Semiconductor Thermal Measurement And Management Symposium (SEMITHERM), Dallas, TX, USA, March 14-16, 2006, pp. 173-177. https://doi.org/10.1109/STHERM.2006.1625224

[25] Poppe, A., Lasance, C. J. M. "Standardization of LED Thermal Characterization", In: Lasance C., Poppe A. (eds.) Thermal Management for LED Applications, Solid State Lighting Technology and Application Series, Vol. 2, Springer, New York, USA, 2013, pp. 197-264.

https://doi.org/10.1007/978-1-4614-5091-7_6

[26] Szalai, A., Szabo, T., Horvath, P., Timar, A., Poppe, A. "Smart SSL: Application of IoT/CPS design platforms in LED-based streetlighting luminaires", In: 2016 IEEE Lighting Conference of the Visegrad Countries (Lumen V4), Karpacz, Poland, Sept. 13-16, 2016, pp. 1-6. https://doi.org/10.1109/LUMENV.2016.7745518

[27] Hegedus, J., Hantos, G., Poppe, A. "Embedded multi-domain LED model for adaptive dimming of streetlighting luminaires", In: 2016 22nd International Workshop on Thermal Investigations of ICs and Systems (THERMINIC), Budapest, Hungary, Sept. 21-23, 2016, pp. 208-212. https://doi.org/10.1109/THERMINIC.2016.7749053

[28] Szekely, V. "Identification of RC networks by deconvolution: chances and limits", IEEE Transactions on Circuits and Systems I: Fundamental Theory and Applications, 45(3), pp. 244-258, 1998. https://doi.org/10.1109/81.662698

[29] Poppe, A., Temesvolgyi, T. "A general multi-domain LED model and its validation by means of AC thermal impedance", In: 29th IEEE Semiconductor Thermal Measurement and Management Symposium (SEMI-THERM), San Jose, CA, USA, March 17-21, 2013, pp. 137-142. https://doi.org/10.1109/SEMI-THERM.2013.6526818

[30] Lang, N., Saak, J., Stykel, T. "Balanced truncation model reduction for linear time-varying systems", Mathematical and Computer Modelling of Dynamical Systems, 22(4), pp. 267-281, 2016. https://doi.org/10.1080/13873954.2016.1198386 